\title{
Influence de l'enherbement viticole sur les Carabidae (Coleoptera) et intérêt potentiel pour le contrôle de certains ravageurs de la vigne
}

\author{
Gaël Petremand ${ }^{(1,3)}$, Dominique Fleury ${ }^{(2)}$, Emmanuel Castella ${ }^{(1)}$, Nicolas Delabays ${ }^{(3)}$ \\ (1) Université de Genève. Institut des Sciences de l'Environnement. Boulevard Carl-Vogt, 66. CH-1211 Genève (Suisse). \\ (2) République et Canton de Genève. Département de l'Environnement, des Transports et de l'Agriculture (DETA). Direction \\ Générale de l'Agriculture et de la Nature (DGAN). Service de l'Agronomie (SAgr). 109, chemin Pont-du-Centenaire. CH- \\ 1228 Plan-les-Ouates (Suisse). E-mail : Dominique.Fleury@etat.ge.ch \\ ${ }^{(3)}$ hepia, HES-SO/Genève. Institut Terre Nature Environnement. Route de Presinge, 150. CH-1254 Jussy (Suisse).
}

Reçu le 1 octobre 2015, accepté le 14 janvier 2016.

Description du sujet. Cet article traite des populations de carabes observés en vignobles.

Objectifs. Les objectifs sont d'évaluer l'impact de différents modes de gestion de l'enherbement viticole sur les populations de carabes et leur potentiel en tant que prédateurs de certains ravageurs de la vigne.

Méthodes. Deux vignobles, caractérisés chacun par différentes gestions de l'enherbement, situés dans le canton de Genève (Suisse), ont été échantillonnés à l'aide de 60 pièges à fosse pour différentes gestions de l'interligne. Les observations ont été effectuées de mars à octobre 2014 et une identification botanique a eu lieu en juin.

Résultats. Au total, ce sont 951 carabes appartenant à 36 espèces qui ont été capturés sur les deux vignobles. Un sol nu, pauvre en espèces végétales et désherbé avec des herbicides, diminue l'abondance des carabes de moitié vis-à-vis d'un enherbement spontané. Un enherbement composé d'une flore spontanée, comparé à des engazonnements, favoriserait les populations de carabes en période de croissance de la vigne en termes de diversité et d'abondance.

Conclusions. La phénologie de certaines espèces de carabes coïncide avec celle de certains ravageurs viticoles. Néanmoins, les espèces les plus fréquemment piégées ont un régime alimentaire omnivore et présenteraient un faible potentiel de prédation. Mots-clés. Agrobiodiversité, viticulture, lutte biologique, ennemis naturels, Carabidae, Suisse.

Ground cover influence on Carabidae (Coleoptera) populations and potential interest for bio-control of some vineyards pests

Description of the subject. This paper deals with carabid populations within vineyards.

Objectives. The objectives were to evaluate the effects of ground cover management on carabid populations and to understand the potential of these beetles as predators of some grapevine pests.

Method. Two vineyards, located in Geneva (Switzerland), were sampled with 60 pitfall traps. Observations were carried out from March to October 2014 and a botanical survey was carried out in June.

Results. In total, 951 carabids belonging to 36 species were captured in the two vineyards. Bare ground and poor plant species treated with herbicides reduced the abundance of the ground beetles. On the other hand, ground covered with spontaneous vegetation, rather than a mixture of seeds, seemed to favor ground beetles throughout the sampling period.

Conclusions. The peak activity of the potential predatory beetles corresponded to the occurrence of some vine pests. However, the most common carabid species captured had an omnivorous diet and a low potential for pest predation on viticulture.

Keywords. Agrobiodiversity, viticulture, biological control, natural enemies, Carabidae, Switzerland.

\section{INTRODUCTION}

En Suisse, plusieurs études ont été menées ces dernières années sur les différentes méthodes d'enherbements viticoles d'un point de vue agronomique (Delabays et al., 2000 ; Delabays et al., 2006 ;Spring et al., 2006) et floristique (Clavien et al., 2006 ; Fortier et al., 2010).
Cependant, aucune d'entre elles n'ont abordé leur impact sur l'entomofaune.

Les carabes (Carabidae : Coleoptera), qui comptent près de 2700 espèces en Europe (Kromp, 1999) et plus de 520 en Suisse (Luka et al., 2009), font partie de la faune épigée du sol, bien que beaucoup d'espèces puissent voler (Lovei et al., 1996). Les carabes adultes 
sont généralement considérés comme étant prédateurs et/ou omnivores (Lovei et al., 1996 ; Kromp, 1999 ; Kotze et al., 2011). Leurs proies sont très diverses en fonction des espèces et au sein d'une même espèce, les imagos étant souvent opportunistes. Ils se nourrissent préférentiellement de certains pucerons, de chenilles de lépidoptères, d'œufs, larves et pupes de diptères, d'œufs et larves de coléoptères et de limaces (Holland et al., 2000). Les espèces omnivores (appartenant principalement aux sous-familles des Harpalinae et des Pterostichinae) consomment majoritairement de la matière végétale (feuilles, fruits, pollen et graines) (Kromp, 1999). Le régime alimentaire des larves est très mal connu, mais il semblerait qu'elles soient plus carnivores que les adultes avec un éventail de proies moins étendu (Lovei et al., 1996).

Dans l'optique d'une lutte biologique par conservation, visant à favoriser une régulation naturelle des populations de ravageurs en améliorant les habitats de leurs ennemis naturels (Ehler, 1998), les carabes possèdent, grâce à leur régime alimentaire, un rôle important de régulation des ravageurs dans les agroécosystèmes cultivés (Kromp, 1999). Par ailleurs, certaines espèces de carabes phytophages pourraient même être de bons régulateurs d'adventices dans les cultures (Bohan et al., 2011).

En vergers et vignobles, la couverture végétale du sol est considérée comme ayant un effet bénéfique sur les communautés d'arthropodes, en particulier certains parasitoïdes (Ichneumonidae, Braconidae, Chalcididae) et prédateurs (araignées, Carabidae, Staphylinidae) des ravageurs (Atlieri et al., 1985). Selon Garcin et al. (2004), la diversité des carabes en vergers est probablement plutôt liée au couvert végétal de l'interligne et à son entretien (fauché ou non) plutôt qu'au mode de culture (production intégrée ou biologique). En Suisse, Genini (2000) a montré que les carabes étaient aussi diversifiés et plus abondants dans les vignes que dans les milieux avoisinants (naturels et rudéraux) et Trivellone et al. (2013) ont recensé la faune carabique des vignobles tessinois donnant un bon aperçu des principales espèces qui se développent en milieu viticole. Au Canada, Goulet et al. (2004) ont mis en évidence que les espèces dominantes étaient différentes suivant la nature du sol du vignoble (argileux ou graveleux-sableux). Malgré leur potentiel de prédation sur certains ravageurs, les variations de composition et d'abondance des carabes induites par diverses gestions de l'interligne des vergers et des vignobles n'ont jamais été étudiées.

La présente étude vise à :

- caractériser la diversité floristique des enherbements viticoles en fonction des différents modes de gestion de l'interligne,

- comparer la faune des Carabidae présente dans les différents enherbements,
- évaluer, selon leur phénologie et leur abondance, le potentiel de prédation des principales espèces piégées sur les principaux ravageurs de la vigne.

Deux questions principales guideront cette recherche: Quel type d'enherbement favorise le plus les carabes au sein de vignobles? Les principales espèces piégées sont-elles utiles pour la régulation des ravageurs viticoles?

\section{MATÉRIEL ET MÉTHODES}

\subsection{Caractéristiques des parcelles}

L'échantillonnage a eu lieu dans deux communes du canton de Genève (Suisse), Soral (SO) et Bernex (BE), dans deux parcelles présentant chacune différents enherbements. Ces deux parcelles ont été choisies pour comparer en leur sein des modes de gestion de l'enherbement différents et non pas pour effectuer une répétition ou une comparaison entre les parcelles. La région d'étude se caractérise par un climat semicontinental avec une température moyenne annuelle de $10,5^{\circ} \mathrm{C}$ et une quantité moyenne de précipitations d'environ $1000 \mathrm{~mm}$ par an. Les deux parcelles sont distantes d'environ $5 \mathrm{~km}$.

Le site de SO (E 490'930/N 110'880), en bordure de la rivière Laire (frontière franco-suisse), est entouré d'un environnement diversifié (forêt, rivière, vignes, grandes cultures). La parcelle, très en pente, repose sur un sol riche calcaire peu profond recouvrant un substrat morainique (dépôts d'origine glaciaire) très caillouteux. Une partie de la parcelle est conduite en gestion conventionnelle avec Désherbage (D) à l'aide d'un herbicide (glyphosate) utilisé deux fois par année sur l'ensemble de la surface. L'autre partie, en production intégrée $(\mathrm{PI})$, présente une flore spontanée (S) sur l'ensemble des interlignes. Elle a été fauchée à trois reprises en 2014 et désherbée uniquement sous le rang à deux reprises à l'herbicide (glyphosate). La lutte par confusion sexuelle a été pratiquée contre les vers de la grappe (cochylis et eudémis) sur l'ensemble de la parcelle. En 2014, des traitements fongiques ont été appliqués (matières actives et nombre d'applications) : folpet (9), soufre mouillable (5), fosétyl-AL (3), quinoxyfen (3), myclobutanil (2), cuivre (2), iprovalicarbe (1), difénoconazole (1) et fénamidone (1).

Le site de BE (E 493'910/N 113'730) est entièrement entouré de vignobles. La parcelle, en pente très faible, repose également sur un sol riche en calcaire. Le substrat de la partie supérieure est très argileux, alors qu'il est plutôt caillouteux dans la partie inférieure. Ces ceps font partie de la station viticole expérimentale de la République de Genève; elles 
sont conduites en PI et six mélanges d'enherbement y ont été semés en automne 2011 pour comparer leur évolution dans le temps (emprise au sol, présence des adventices) et leur impact agronomique (concurrence sur l'alimentation hydrique et azotée) en lien avec la vigne. Quatre répétitions de $40 \mathrm{~m}^{2}$ de chaque mélange ainsi que d'un témoin composé de la végétation spontanée ont été réalisées sur huit interlignes. Compte tenu de l'impossibilité d'étudier l'entomofaune sur chacun des mélanges, nous avons choisi d'inventorier les deux enherbements les plus différents (dominance d'espèces caractéristiques du mélange, faible fréquence des adventices) au début du mois de mars 2014 : Schweizer Lenta ${ }^{\circledR}$ (L) composé de graminées (Annexe 1) et Schweizer Neue ${ }^{\circledR}(\mathrm{N})$ ou « Biodiversité viticole » (Annexe 1) ; le témoin (T) était caractérisé par une végétation spontanée. Cette parcelle, conduite en PI, a été fauchée à quatre reprises. Un désherbage sous le rang a été pratiqué à deux reprises durant la saison à l'herbicide (glufosinate, glyphosate) et une lutte par confusion sexuelle a été mise en place contre les vers de la grappe. Des traitements fongiques ont été effectués en 2014 (matières actives et nombre d'applications) : soufre mouillable (11), folpet (11), hydroxyde de cuivre (6), spiroxamine (3), tébuconazole (2), métalaxyl M (2), cymoxanil (2), fosétyl-Al (2), mandipropamide (2), iprovalicarbe (1), quinoxyfen (1), cyprodinil (1), fludioxonil (1), fenpropidine (1) et métrafénone (1).

\section{2. Échantillonnage}

Relevés botaniques. A SO, des relevés qualitatifs ont été menés le 16 juin 2014 sur trois interlignes de chaque type de gestion de l'interligne $(\mathrm{D}, \mathrm{S})$. Pour chaque espèce, un pourcentage de recouvrement a été estimé. Sur cette parcelle, des relevés quantitatifs n'étaient pas nécessaires étant donné la nature très différente des deux types de gestion de l'enherbement.

À $\mathrm{BE}$, les relevés de végétation ont été effectués les 5 et 12 juin 2014 par une méthode de relevé linéaire dite des points quadrats (Levy et al., 1933). Deux transects de $2 \mathrm{~m}$ ont été effectués dans trois des quatre réplicats de chaque type d'enherbement. Tous les $10 \mathrm{~cm}$ le long du transect, à l'aide d'une tige métallique, sont relevées les espèces touchant la tige. Une espèce est relevée une seule fois par point, même si plusieurs individus touchent la tige. Cette technique permet d'estimer les fréquences spécifiques de chaque espèce. Pour chaque enherbement, six transects et 126 points de relevé ont été effectués. Les espèces ont été déterminées à l'aide des flores de Aeschimann et al. (1994) et Lauber et al. (2012).

Relevés entomologiques. Les piégeages des carabes ont été effectués au moyen de pièges à fosse, méthode largement utilisée pour échantillonner la faune épigée (Greensdlade, 1964). Des pots en plastique de $10 \mathrm{~cm}$ de diamètre ont été enterrés dans le sol en veillant à ce que leur bord supérieur soit au niveau de la surface du sol. Les pots ont été remplis au tiers de leur volume avec de l'alcool à $70^{\circ}$. Les pots ont été couverts par un toit pour empêcher les précipitations d'y pénétrer.

$\mathrm{Au}$ total, 60 pièges ont été posés et sont restés installés dans les vignobles quatre jours par mois (de mars à septembre), ce qui représente 28 jours d'échantillonnage sur sept relevés (un par mois). A SO, en accord avec certains principes énoncés par Kotze et al. (2011) pour l'échantillonnage par piège à fosse, nous avons disposé 12 pièges par zone $(\mathrm{S}$ et $\mathrm{D})$ groupés par quatre en carré à $2,50 \mathrm{~m}$ les uns des autres. À BE, le principe est similaire, trois groupes de quatre pièges ont été disposés dans chaque type d'enherbement ( $\mathrm{T}$, $\mathrm{L}, \mathrm{N})$.

Les individus capturés ont été triés manuellement pour en extraire les carabes. Ces derniers ont été déterminés au niveau de l'espèce à l'aide de Trautner et al. (1987), Müller-Motzfeld (2004) et Luff et al. (2007). Les identifications ont été vérifiées par Alain Ducommun (Insecta, Neuchâtel) et Henryk Luka (FiBL, Frick). La nomenclature utilisée suit Löbl et al. (2003).

\subsection{Analyse des données}

Botanique. Les richesses spécifiques de chaque type de gestion de l'interligne ont été calculées pour les deux sites expérimentaux. Les fréquences de chaque espèce à $\mathrm{BE}$ ont été calculées afin de faire ressortir les espèces dominantes et de cibler les espèces encore présentes après le semis de 2011.

Carabes. L'abondance des carabes dans les pièges est une mesure de densité d'activité (Kotze et al., 2011). L'abondance totale (somme des individus piégés), la richesse spécifique (nombre d'espèces), la diversité de Shannon (mesure de diversité qui intègre l'abondance de chaque espèce) et la richesse raréfiée ainsi que son erreur standard (ES) (fonction «rarefy», package «vegan », logiciel R) (Heck et al., 1975) ont été calculées pour chaque type de gestion de l'interligne (D, S, T, L, N). Selon la loi normale, les valeurs de richesse raréfiée sont statistiquement différentes si elles ne se chevauchent pas après soustraction ou addition de l'ES x 1,96. Les enherbements ont été comparés par le test non paramétrique des rangs signés de Wilcoxon (seuil de $5 \%$ ) sur les abondances des espèces (Hollander et al., 1973) à l'aide du logiciel de statistiques Minitab 17. Une sélection des espèces dominantes (selon leur abondance relative) et constante dans le temps (selon leur fréquence dans les relevés) a également été réalisée (Garcin et al., 2004). 
Une espèce est dominante si elle représente $>50 \%$ des individus capturés au sein du même enherbement. Une espèce est constante si elle est présente dans 25 à $50 \%$ des relevés et accessoires si elle apparait dans $\leq 25 \%$ des relevés. La phénologie des espèces sélectionnées a été ensuite illustrée graphiquement afin de faire un lien avec les pics de certains ravageurs de la vigne.

\section{RÉSULTATS}

\subsection{Botanique}

Les relevés botaniques qualitatifs effectués à SO révèlent une richesse de 16 espèces en $\mathrm{D}$ (avec un recouvrement du sol de $10 \%$, sous forme d'un mulch partiellement sec) et 40 espèces en $\mathrm{S}$ avec un recouvrement du sol de $80 \%$. Cinq espèces ont un recouvrement $\geq 1 \%$ en $\mathrm{D}$ (Arenaria serpyllifolia, Bromus sterilis, Cardamine hirsuta, Lollium perenne, Hordeum murinum) et 15 espèces en $S$ parmi lesquelles deux espèces sont largement dominantes : le néophyte Erigeron annuus (20\%) et Trifolium repens (35\%).

À BE (Tableau 1), l'enherbement le plus riche est $\mathrm{N}$ avec 22 espèces (contre 15 et 16 pour $\mathrm{T}$ et $\mathrm{L}$ ). Dans le témoin (T), la flore est dominée par T. repens ( $\geq 85 \%)$, suivent d'autres adventices des vignobles, principalement Convolvulus arvensis, Cerastium fontanum, Picris hieracioides et Taraxacum offcinale, ainsique d'autres espècespouvant provenirdesmélanges semés à proximité, notamment L. perenne et Prunella vulgaris. L'enherbement $\mathrm{L}$ est également largement dominé par T. repens ( $\leq 85 \%)$, ainsi que par Festuca rubra $(85 \%)$ et Poa pratensis $(60 \%)$, deux espèces semées dans le mélange d'origine. L'enherbement $\mathrm{N}$ est principalement composé de T.repens $(\geq 60 \%)$, P. vulgaris $(\leq 60 \%)$, Sanguisorba minor $(\leq 55 \%)$, les deux dernières ayant été originellement semées. Deux autres espèces présentes dans le mélange montrent une fréquence importante : Lotus corniculatus (35\%) et Medicago lupulina (20\%). Les quatre principales adventices des vignobles, Convolvusus arvensis, $L$. perenne, $T$. officinale et $T$. repens, ont les fréquences les plus basses en $\mathrm{N}$.

\subsection{Carabes}

Comparaison générale des enherbements. Au total, entre mars et septembre 2014, ce sont 951 individus appartenant à 36 espèces qui ont été capturés sur les deux sites. À SO, 19 espèces ont été répertoriées contre 31 à $\mathrm{BE}$. En termes de richesse et de diversité de Shannon (Tableau 2), D et $\mathrm{S}$ montrent une différence, l'enherbement $S$ étant plus riche et plus diversifié, même si les richesses raréfiées ne sont pas significativement différentes selon l'ES. En S, le
Tableau 1. Fréquence des espèces (en \%) dans les relevés botaniques par point-quadrat effectués à Bernex (BE) les 5 et 12 juin 2014 dans les différents enherbements - Species frequency (in \%) in botanical surveys using point-quadrat method done at Bernex (BE) on 5 and 12 June 2014 in the different ground cover vegetations.

\begin{tabular}{|c|c|c|c|}
\hline Espèces & $\mathbf{T}$ & $\mathbf{L}$ & $\mathbf{N}$ \\
\hline Belis perennis L. & 6 & 2 & \\
\hline Cerastium arvensis L. s.str. & & 2 & 5 \\
\hline Cerastium fontanum Baumg. s.1. & 34 & & \\
\hline Clinopodium vulgare $\mathrm{L}$. & & 3 & \\
\hline Convolvulus arvensis $L$. & 36 & 44 & 29 \\
\hline Daucus carota L. & & & 3 \\
\hline Dianthus carthusianorum L. s.l.** & & & 4 \\
\hline Festuca rubra aggr.* & 4 & 85 & \\
\hline Geranium dissectum L. & & 6 & 1 \\
\hline Geranium pyrenaicum Burm. f. & 7 & 1 & 6 \\
\hline Lolium perenne $L$. & 22 & 12 & 5 \\
\hline Lotus corniculatus L.** & & & 35 \\
\hline Medicago lupulina L.** & 3 & & 20 \\
\hline Ononis spinosa L. s.1.** & & & 1 \\
\hline Picris hieracioides L. s.l. & 18 & 1 & 10 \\
\hline Plantago lanceolata L. & 2 & 2 & 9 \\
\hline Poa annua L. & & & 5 \\
\hline Poa pratensis L.* & 24 & 60 & \\
\hline Polygonum aviculare L. & & & 1 \\
\hline Potentilla verna auct.** & & & 6 \\
\hline Prunella vulgaris L.** & 23 & & 59 \\
\hline Sanguisorba minor Scop. s.str. ** & & 2 & 54 \\
\hline Taraxacum officinale aggr. & 13 & 7 & 4 \\
\hline Trifolium repens $L$. & 86 & 84 & 62 \\
\hline Veronica filiformis $\mathrm{Sm}$. & 2 & & 2 \\
\hline Veronica triphyllos L. & 2 & & 2 \\
\hline Vicia sativa L. s.1. & 2 & 2 & 4 \\
\hline Richesse & 16 & 15 & 22 \\
\hline \multicolumn{4}{|c|}{$\begin{array}{l}\text { T : témoin - control; * : espèces du mélange Schweizer } \\
\text { Lenta }{ }^{\circledR}(\mathrm{L}) \text { semées en automne } 2011-\text { species from the } \\
\text { Schweizer Lenta@ }(\text { L) mix sown in autumn } 2011 ; * * \text { : espèces } \\
\text { du mélange Schweizer Neue }{ }^{\circledR}(\mathrm{N}) \text { semées en automne } \\
2011 \text { - species from the Schweizer Neue@ }(N) \text { mix sown in } \\
\text { autumn } 2011 \text {; en gras, les adventices fréquentes des vignobles } \\
\text { de la Suisse occidentale }- \text { in bold the frequent weeds in } \\
\text { vineyards of West Switzerland (Clavien et al., 2006). }\end{array}$} \\
\hline
\end{tabular}


Tableau 2. Indices de comparaison des populations de carabes entre les types de gestion de l'interligne à Soral (SO) et Bernex (BE) - Comparison of Carabidae populations in several ground cover vegetations management in Soral (SO) and Bernex (BE).

\begin{tabular}{llllll}
\hline Parcelle & Type d'enherbement & $\begin{array}{l}\text { Nombre } \\
\text { d'individus }\end{array}$ & Richesse & $\begin{array}{l}\text { Indice de diversité } \\
\text { de Shannon }\end{array}$ & $\begin{array}{l}\text { Richesse raréfiée } \\
\text { (erreur standard) }\end{array}$ \\
\hline Soral & D (Végétation désherbée) & 44 & 12 & 1,89 & $11,45(0,66)$ \\
& S (Végétation spontanée) & 101 & 16 & 2,25 & $11,78(1,34)$ \\
\hline \multirow{2}{*}{ Bernex } & T (Témoin) & 362 & 20 & 2,18 & $15,46(1,49)$ \\
& L (Schweizer Lenta) & $143^{*}$ & 20 & 1,85 & $17,06(1,40)$ \\
& N (Schweizer Neue) & 276 & 24 & 1,91 & $16,21(1,79)$ \\
\hline
\end{tabular}

La richesse raréfiée a été calculée avec $: \mathrm{n}=40$ individus pour $\mathrm{D}$ et $\mathrm{S}, \mathrm{n}=100$ individus pour $\mathrm{T}, \mathrm{L}$ et $\mathrm{N}$. $\mathrm{n}=12$ pièges par type d'enherbement - Rarefied richness was calculated with: $n=40$ individuals for $D$ and $S, n=100$ individuals for T, L and N. $n=12$ traps per type of ground cover vegetation; * : significativement différent avec $\mathrm{T}$ et $\mathrm{N}-$ significatively different with $T$ and $N$.

nombre d'individus est deux fois supérieur à $\mathrm{D}$, mais de manière non significative (test de Wilcoxon: $p=$ $0,06)$. Sur le site BE (Tableau 2), les enherbements se différencient surtout en termes d'abondance avec un total d'individus très faible pour $\mathrm{L}$ et significativement plus faible que pour les deux autres enherbements $(\mathrm{N}$ : $p=0,02 ; \mathrm{T}: p=0,005)$. Les abondances d'espèces ne sont pas significativement différentes entre $\mathrm{T}$ et $\mathrm{N}$ $(p=0,2)$. La diversité de Shannon est plus élevée en T, probablement à cause d'une dominance relativement moins importante de Harpalus rufipes (Tableau 4). La richesse spécifique est la plus grande dans $\mathrm{N}(24$ espèces), mais les richesses raréfiées ne se différencient pas statistiquement entre les enherbements.

\subsection{Composition spécifique des enherbements}

L'enherbement $S$ possède trois espèces constantes et six espèces accessoires contre respectivement deux et quatre en D (Tableau 3). Deux des trois espèces constantes en $\mathrm{S}$ le sont également en $\mathrm{D}$ et sont dominantes : Amara aenea et Ophonus azureus.

À BE (Tableau 4), l'enherbement $\mathrm{T}$ possède le plus d'espèces constantes (8) et $\mathrm{N}$ le plus d'espèces accessoires (7). Le mélange L possède peu d'espèces constantes ou accessoires et une espèce largement dominante (H.rufipes: $55 \%$ des effectifs). Cinq espèces en $\mathrm{T}$ (A.aenea, H.rufipes, Harpalus dimidiatus, Harpalus tardus et $O$.azureus), trois en $\mathrm{L}$ (A. aenea, Harpalus rubripes et H.rufipes) et quatre en N (A. aenea, Anchomenus dorsalis, H. dimidiatus et $H$.rufipes) ont été observées comme constantes et dominantes.

\subsection{Phénologie des espèces principales}

Il existe une nette différence des dynamiques d'abondance des espèces entre $\mathrm{D}$ et $\mathrm{S}$ (Figure 1). Un seul pic d'abondance se dessine en $\mathrm{D}$ au mois de juin, contrairement à $\mathrm{S}$ où un premier pic d'abondances est net en mai et un deuxième en juillet-aout. Amara aenea domine clairement en D (45\% des individus, tableau 3) avec un pic en juin qui correspond à l'arrivée d'individus immatures sortant de la nymphose.

Sur le site BE, l'enherbement $T$ (Figure 2) possède une communauté carabique nombreuse et constante comme le montrent les abondances cumulées des espèces. Un premier pic a lieu en juin avec l'augmentation des abondances de A. aenea et Ophonus azureus et un deuxième en aout dû à l'augmentation importante de H.rufipes. Dans les mélanges L et $\mathrm{N}$ (Figure 2), le deuxième pic est également observé. Les mois précédents, les abondances cumulées sont relativement constantes sans pic au mois de juin.

\section{DISCUSSION}

Parmi les 36 espèces de Carabidae capturées dans les deux sites d'étude, six d'entre elles sont xérophiles (Luka et al., 2009) : Amara bifrons, Callistus lunatus, Harpalus honestus, Ophonus ardosiacus, Ophonus azureus et Ophonus schaubergerianus. La vigne peut donc abriter certaines espèces habituellement associées à des milieux secs. Trois espèces présentant un intérêt pour la conservation de la biodiversité (sur la liste rouge suisse), parmi lesquelles deux espèces sont également xérophiles, ont été collectées par Pétremand (2015) sur le site de l'étude : Agonum nigrum, Brachinus sclopeta et Notiophilus aestuans.

Les relevés botaniques à SO montrent que les traitements herbicides induisent une grande différence de recouvrement et de richesse floristique. Cette différence a pour conséquence une diminution du nombre total d'individus de la communauté des Carabidae et des principales espèces du site en D. Cette observation confirme celles d'Altieri et al. (1985), selon lesquels la présence d'un couvert végétal favorise certains auxiliaires (araignées, hyménoptères parasitoïdes). Les espèces dominantes et constantes appartiennent 
Tableau 3. Abondance relative (ab. rel.) et constance des espèces de Carabidae présentes dans les deux types d'enherbement, Désherbé (D) et Spontané (S), à Soral (SO) - Relative abundance (ab. rel.) and constancy of species present in both types of management "Désherbé" (D) and "Spontané" (S) in Soral (SO).

\begin{tabular}{|c|c|c|c|c|c|}
\hline \multirow[t]{2}{*}{ Espèce } & \multicolumn{2}{|l|}{$\mathbf{S}$} & \multicolumn{2}{|l|}{ D } & \multirow{2}{*}{$\begin{array}{l}\text { Régime } \\
\text { alimentaire }\end{array}$} \\
\hline & Ab. rel. (\%) & Constance & Ab. rel. (\%) & Constance & \\
\hline Amara aenea (De Geer, 1774) & 8 & $\mathbf{C}$ & 45 & $\mathbf{C}$ & $\mathrm{O}$ \\
\hline Amara bifrons (Gyllenhal, 1810) & & & 2 & & G \\
\hline Amara lucida (Duftschmid, 1812) & & & 2 & & $\mathrm{O}$ \\
\hline Bembidion lampros (Herbst, 1784) & 1 & & & & $\mathrm{P}$ \\
\hline Callistus lunatus Fabricius, 1775 & 1 & & 7 & $\mathbf{A}$ & $\mathrm{P}$ \\
\hline Carabus auratus L., 1761 & 1 & & & & $\mathrm{P}$ \\
\hline Carabus coriaceus L., 1758 & 1 & & & & $\mathrm{P}$ \\
\hline Harpalus affinis (Schrank, 1781) & & & 2 & & $\mathrm{O}$ \\
\hline Harpalus dimidiatus (P. Rossi, 1790) & 9 & $\mathbf{A}$ & 7 & A & $\mathrm{O}$ \\
\hline Harpalus distinguendus (Duftschmid, 1812) & 4 & A & 2 & & $\mathrm{O}$ \\
\hline Harpalus rubripes (Duftschmid, 1812) & 18 & C & 2 & & $\mathrm{O}$ \\
\hline Harpalus rufipes (De Geer, 1774) & 5 & A & 7 & A & $\mathrm{O}$ \\
\hline Harpalus tardus (Panzer, 1796) & 2 & & 9 & $\mathbf{A}$ & $\mathrm{O}$ \\
\hline Microlestes maurus (Sturm, 1827) & 2 & & & & $\mathrm{P}$ \\
\hline Ophonus ardosiacus (Lutshnik, 1922) & 3 & A & & & $\mathrm{O}$ \\
\hline Ophonus azureus (Fabricius, 1775) & 29 & $\mathbf{C}$ & 11 & $\mathbf{C}$ & $\mathrm{O}$ \\
\hline Ophonus schaubergerianus (Puel, 1937) & 8 & $\mathbf{A}$ & & & $\mathrm{O}$ \\
\hline Parophonus maculicornis (Duftschmid, 1812) & 1 & & & & $\mathrm{O}$ \\
\hline Poecilus cupreus L., 1758 & 8 & A & 2 & & $\mathrm{P}$ \\
\hline Total & 100 & & 100 & & \\
\hline \multicolumn{6}{|c|}{$\begin{array}{l}\mathrm{C}: \text { espèces constantes, présentes dans }>50 \% \text { des relevés; A : espèces accessoires, présentes dans }>25 \% \text { des relevés. En gras, les } \\
\text { pourcentages }>5 \% \text { définissant les espèces dominantes. Régime alimentaire : O, espèces omnivores, P, espèces prédatrices strictes, G, } \\
\text { espèces granivores. } \mathrm{n}=12 \text { pièges par type d'enherbement }- \text { The relative abundance is the percentage of individuals of a species on the } \\
\text { total. Constancy is defined by a C for constant species present in }>50 \% \text { of the surveys and an A for the accessorie species present in } \\
>25 \% \text { of the surveys. In bold percentages }>5 \% \text { defining the dominant species. The diet is given by an O for omnivorous species, P for }\end{array}$} \\
\hline
\end{tabular}

aux sous-familles des Harpalinae (Harpalus ssp. et Ophonus ssp.) et des Pterostichinae (Amara ssp.) dont les espèces au stade adulte sont considérées comme omnivores, ces espèces font partie des principales espèces identifiées par Trivellone et al. (2013) au Tessin (Suisse). Leur régime alimentaire est principalement composé de graines, mais une prédation opportuniste a également été montrée sur pucerons (Myzus persicae), pupes de diptères (Bactrocera oleae) et chenilles de lépidoptères (Cydia pomonella), notamment pour A. aenea (uniquement des pucerons) et des espèces du genre Harpalus (Garcin et al., 2006). La végétation spontanée favorise plus d'espèces constantes et accessoires qu'un sol nu et donc un potentiel de prédation supérieur sur divers ravageurs, malgré l'arrivée d'individus immatures d'A. aenea en juin dans D qui peuvent représenter un potentiel de prédation. Amara aenea, xérophile, a été l'espèce la plus piégée $(25 \%$ des individus collectés) dans 48 vignobles tessinois, elle parait donc très adaptée au milieu viticole et aux perturbations qui y sont liées (Trivellone et al., 2013).

Les enherbements de BE sont qualitativement très différents entre eux d'un point de vue botanique. Les mélanges semés trois ans avant les relevés sont toujours composés d'espèces présentes initialement dans les semis et ayant des fréquences relatives importantes dans les relevés. Une seule espèce est commune et dominante dans les trois enherbements : T. repens. Les quatre adventices principales des vignobles ayant les fréquences les plus basses dans l'enherbement $\mathrm{N}$, ce dernier semble donc être plus efficace que L contre l'expansion des adventices, malgré le bon recouvrement au sol avec le mélange Schweizer Lenta ${ }^{\circledR}$ (Spring et al., 2006). 
Tableau 4. Abondance relative (ab. rel.) et constance des espèces de Carabidae présentes dans les trois enherbements : Témoin $(\mathrm{T})$, Lenta $(\mathrm{L})$ et Neu $(\mathrm{N})$ à Bernex $(\mathrm{BE})$ - Relative abundance $(a b$. rel.) and constancy of Carabidae species in three ground cover vegetation: Témoin $(T)$, Lenta $(L)$ and Neu $(N)$ in Bernex $(B E)$.

\begin{tabular}{|c|c|c|c|c|c|c|c|}
\hline \multirow[t]{2}{*}{ Espèce } & \multicolumn{2}{|l|}{$\mathbf{T}$} & \multicolumn{2}{|l|}{$\mathbf{L}$} & \multicolumn{2}{|l|}{$\mathbf{N}$} & \multirow[t]{2}{*}{ Régime } \\
\hline & $\begin{array}{l}\text { Ab. rel. } \\
(\%)\end{array}$ & Constance & $\begin{array}{l}\text { Ab. rel. } \\
(\%)\end{array}$ & Constance & $\begin{array}{l}\text { Ab. rel. } \\
(\%)\end{array}$ & Constance & \\
\hline Agonum nigrum Dejean, 1828 & & & & & 0,4 & & $\mathrm{P}$ \\
\hline Amara aenea (De Geer, 1774) & 22 & $\mathrm{C}$ & 7,7 & $\mathrm{C}$ & 3,6 & $\mathrm{C}$ & $\mathrm{O}$ \\
\hline Amara bifrons (Gyllenhal, 1810) & 0,3 & & & & & & G \\
\hline Amara communis (Panzer, 1797) & & & & & 0,7 & & $\mathrm{O}$ \\
\hline Amara lucida (Duftschmid, 1812) & 1,7 & A & 0,7 & & & & $\mathrm{O}$ \\
\hline $\begin{array}{l}\text { Anchomenus dorsalis (Pontoppidan, } \\
\text { 1763) }\end{array}$ & 1,9 & $\mathrm{C}$ & 0,7 & & 6,2 & $\mathrm{C}$ & $P$ \\
\hline Badister bullatus (Schrank, 1798) & & & 0,7 & & 1,1 & A & $\mathrm{P}$ \\
\hline Brachinus crepitans (L., 1758) & & & & & 0,4 & & $\mathrm{P}$ \\
\hline Brachinus explodens Duftschmid, 1812 & 3,0 & A & 0,7 & & 0,4 & & $\mathrm{P}$ \\
\hline Bembidion lampros (Herbst, 1784) & 0,8 & & 1,4 & & 0,7 & & $\mathrm{P}$ \\
\hline Bembidion quadrimaculatum (L., 1761) & & & 0,7 & & 0,4 & & $\mathrm{P}$ \\
\hline Callistus lunatus (Fabricius, 1775) & 0,3 & & & & & & $P$ \\
\hline Harpalus affinis (Schrank, 1781) & 2,8 & A & 2,1 & A & 3,6 & A & $\mathrm{O}$ \\
\hline Harpalus anxius/subcylindricus & & & 1,4 & & & & $\mathrm{O}$ \\
\hline Harpalus dimidiatus (P. Rossi, 1790) & 8,6 & $\mathrm{C}$ & 4,2 & $\mathrm{C}$ & 9,4 & $\mathrm{C}$ & $\mathrm{O}$ \\
\hline $\begin{array}{l}\text { Harpalus distinguendus (Duftschmid, } \\
\text { 1812) }\end{array}$ & 3,9 & $\mathrm{C}$ & 0,7 & & 1,8 & $\mathrm{~A}$ & $\mathrm{O}$ \\
\hline Harpalus griseus (Panzer, 1796) & 1,1 & & & & 0,7 & & $\mathrm{O}$ \\
\hline Harpalus honestus (Duftschmid, 1812) & 0,8 & & 2,1 & A & & & $\mathrm{O}$ \\
\hline Harpalus rubripes (Duftschmid, 1812) & 2,5 & $\mathrm{C}$ & 7,0 & $\mathrm{C}$ & 1,4 & A & $\mathrm{O}$ \\
\hline Harpalus rufipes (De Geer, 1774) & 32 & $\mathrm{C}$ & 55 & $\mathrm{C}$ & 52 & $\mathrm{C}$ & $\mathrm{O}$ \\
\hline Harpalus serripes (Quensel, 1806) & & & 0,7 & & 0,4 & & $\mathrm{O}$ \\
\hline Harpalus tardus (Panzer, 1796) & $\mathbf{5 , 8}$ & $\mathrm{C}$ & 4,9 & A & 2,9 & A & $\mathrm{O}$ \\
\hline Microlestes maurus (Sturm, 1827) & 0,3 & & & & 0,4 & & $\mathrm{P}$ \\
\hline Nebria brevicollis (Fabricius, 1792) & 0,3 & & & & 0,4 & & $\mathrm{P}$ \\
\hline Ophonus azureus (Fabricius, 1775) & 9,9 & $\mathrm{C}$ & 2,8 & A & 8,3 & $\mathrm{C}$ & $\mathrm{O}$ \\
\hline $\begin{array}{l}\text { Parophonus maculicornis (Duftschmid, } \\
\text { 1812) }\end{array}$ & 0,8 & & & & 0,4 & & \\
\hline Poecilus cupreus L., 1758 & 1,7 & A & 5,6 & A & 2,9 & A & $\mathrm{P}$ \\
\hline Pterostichus vernalis (Panzer, 1796) & & & & & 0,4 & & $\mathrm{P}$ \\
\hline Tachys bistriatus (Duftschmid, 1812) & & & 0,7 & & 1,1 & A & $\mathrm{P}$ \\
\hline Trechus quadristriatus (Schrank, 1781) & & & 0,7 & & & & $\mathrm{P}$ \\
\hline Total & 100 & & 100 & & 100 & & \\
\hline
\end{tabular}

$\mathrm{C}$ : espèces constantes, présentes dans $>50 \%$ des relevés; $\mathrm{A}$ : espèces accessoires présentes dans $>25 \%$ des relevés. En gras, les pourcentages $>5 \%$ définissant les espèces dominantes ; Régime alimentaire : $O$, espèces omnivores ; $P$, espèces prédatrices strictes ; $\mathrm{G}$, espèces granivores. $\mathrm{n}=12$ pièges par type d'enherbement - The relative abundance is the percentage of individuals of a species on the total. Constancy is defined by a C for constant species present in $>50 \%$ of the surveys and an A for the accessorie species present in $>25 \%$ of the surveys. In bold, percentages $>5 \%$ defining the dominant species. The diet is given by an $O$ for omnivorous species, $P$ for strict predatory species and $G$ for seed-eating species. $n=12$ traps per type ground cover vegetation. 


\section{Zone désherbée}

Densité d'activité cumulée des espèces principales

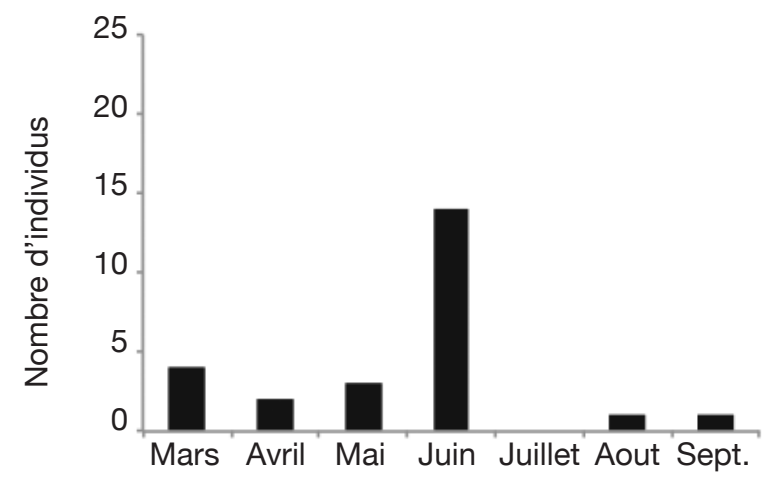

Enherbement spontané

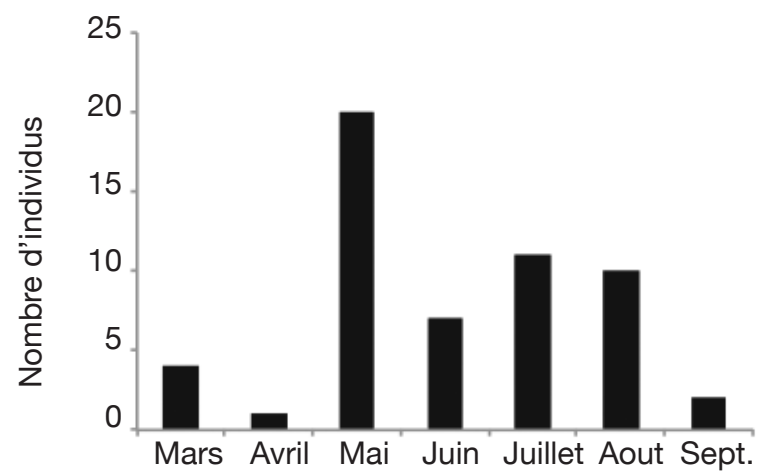

Densité d'activité des espèces contribuant aux pics principaux d'activité

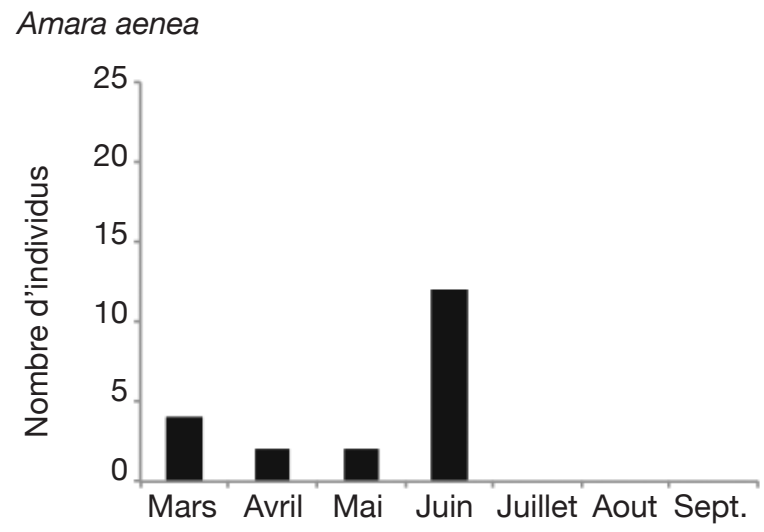

Figure 1. Densités d'activités mensuelles cumulées des espèces principales (dominantes et constantes) de carabes et par espèce contribuant le plus aux principaux pics d'activité dans la zone désherbée et dans la zone ayant un enherbement spontané à Soral - Monthly cumulated activity density of main (dominant and constant) carabid species and monthly activity density of species that most contribute to peaks of activity, in weeded area (D) and in area with spontaneous vegetation $(S)$ in Soral.

L'enherbement L montre une abondance des carabes et un nombre d'espèces constantes et accessoires relativement faible comparativement à $\mathrm{N}$ et $\mathrm{T}$; la dominance d'espèces graminées semble globalement ne pas être très profitable à la communauté carabique. L'indice de diversité de Shannon montre qu'il y a un meilleur équilibre entre les espèces en $\mathrm{T}$ où le plus grand nombre d'individus a été capturé. La phénologie et les abondances des principales espèces
Ophonus azureus
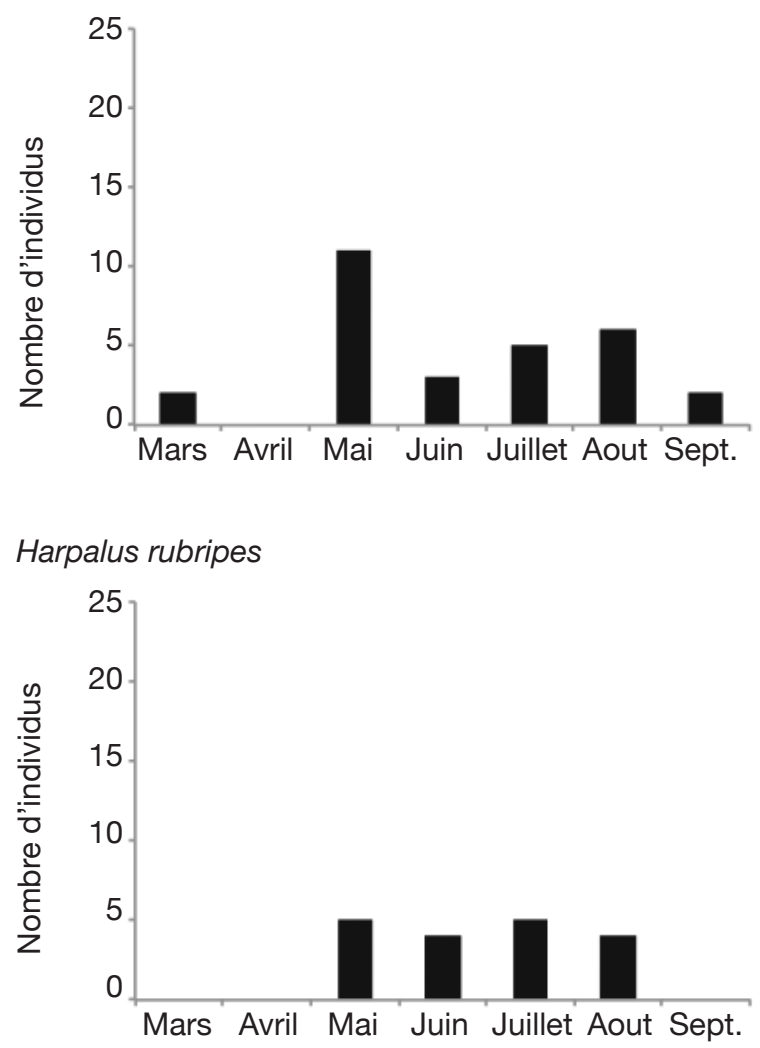

dans ces enherbements mettent en évidence le fait que $\mathrm{T}$ favorise l'occurrence et la constance des espèces, ce qui concorde avec les conclusions de Danne et al. (2010) selon lesquels un enherbement composé de la flore spontanée favorise les arthropodes auxiliaires en viticulture. En 2014, les deux pics d'abondance cumulée coïncident avec les pics de captures des vers de la grappe Eudémis (Lobesia botrana) et Cochylis (Eupoecilia ambiguella) (Pasquier, 2014). Les espèces 
Témoin

Densité d'activité cumulée des espèces principales

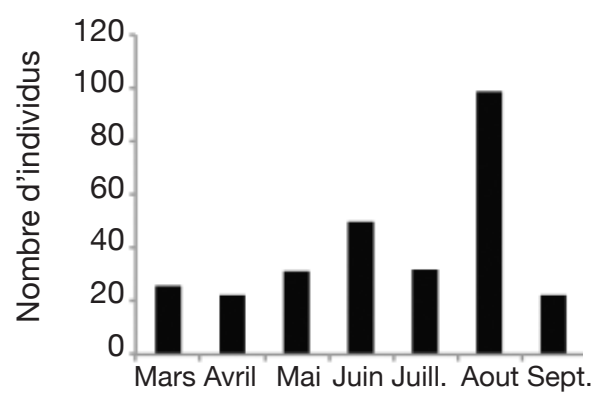

Schweizer Lenta®

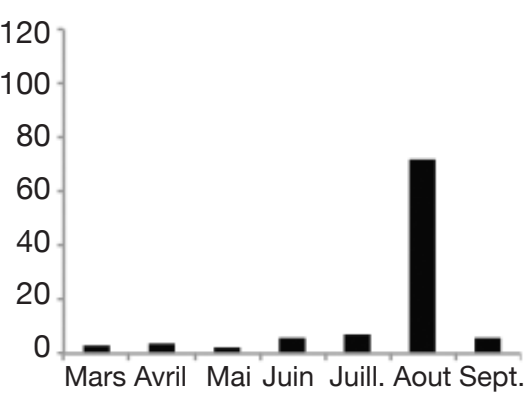

Schweizer Neue $®$

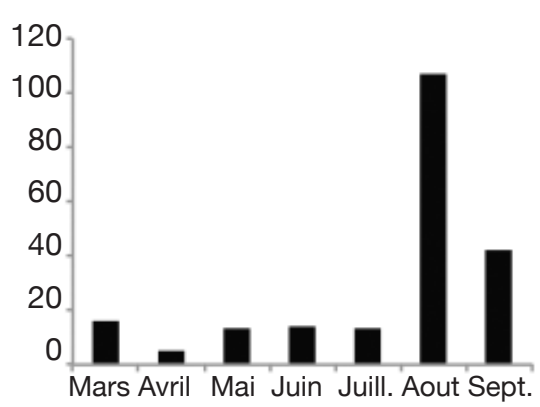

Densité d'activité des espèces contribuant aux pics principaux d'activité

Harpalus rufipes
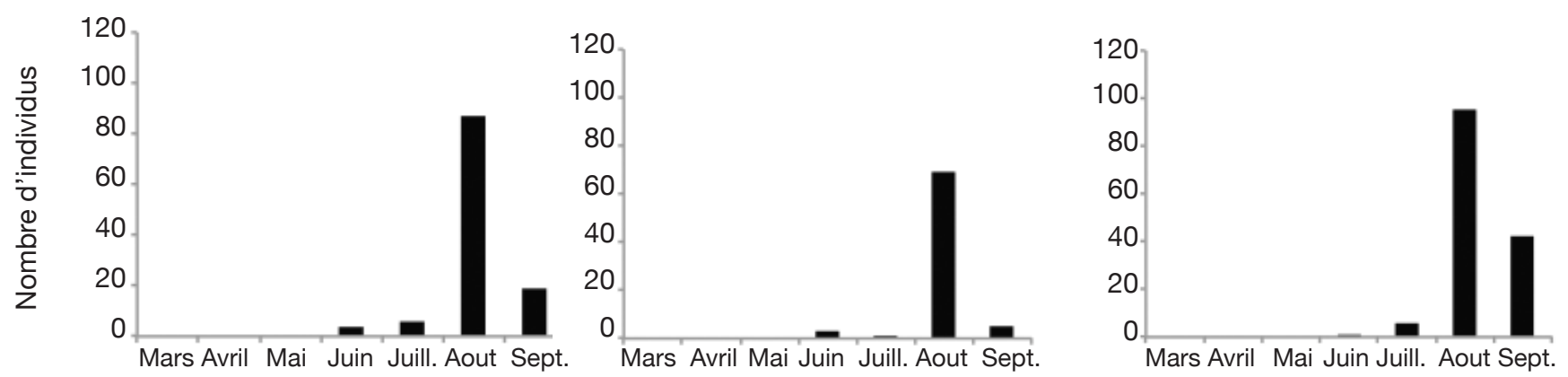

Amara aenea

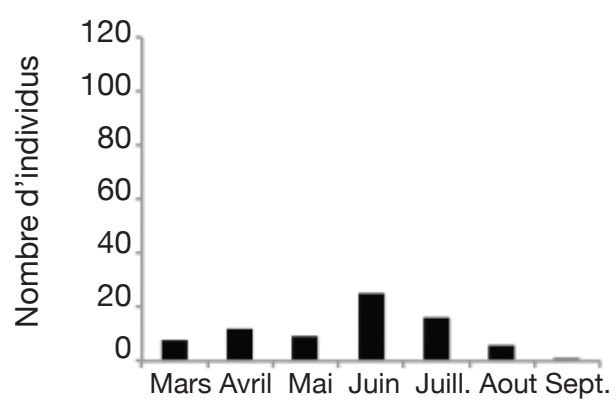

Ophonus azureus
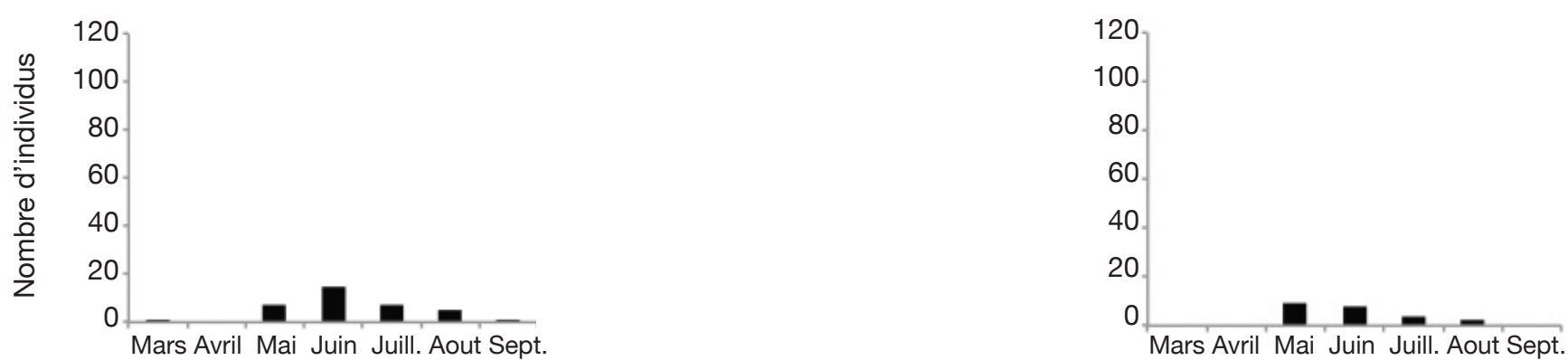

Figure 2. Densités d'activités mensuelles cumulées des espèces principales (dominantes et constantes) de carabes et par espèce contribuant le plus aux principaux pics d'activité dans l'enherbement témoin (T), le mélange botanique Lenta (L) et le mélange botanique Neue $(\mathrm{N})$ à Bernex $(\mathrm{BE})$ - Monthly cumulated activity density of main (dominant and constant) carabid species and monthly activity density of species that most contribute to peaks of activity, in ground cover compound of native vegetation (T), seed mixture Lenta $(L)$ and seed mixture Neue $(N)$ in Bernex $(B E)$. 
du genre Harpalus peuvent potentiellement exercer une prédation sur les larves de ces deux lépidoptères ainsi que sur celles de noctuelles telles que Noctua comes et Phlogophora meticulosa qui ont un long stade de développement larvaire au sol et sur les adventices des vignobles (Hächler et al., 1993). Ces carabes peuvent également consommer des pucerons (ex.: M. persicae ou Aphis pomi) tombés au sol ainsi que des larves et des pupes de Diptères (ex.: Rhagoletis pomonella, Rhagoletis cerasi, Bactrocera oleae ou Drosophila sp.) ayant un stade de développement au sol (Garcin et al., 2006). Le deuxième pic d'abondance des carabes, induit par le nombre élevé de captures de H.rufipes en aout, est aussi observé en L et N. Harpalus rufipes ne semble donc pas être sensible à la nature du couvert végétal ; cette espèce a également été la plus capturée en Valais (Suisse) (Genini, 2000). Elle possède donc un potentiel de prédation important, notamment sur les deuxièmes générations des vers de la grappe durant le mois d'aout où sa densité d'activité est grande dans l'ensemble des enherbements viticoles.

Finalement, malgré ce milieu fortement perturbé (fauches, pesticides, etc.), il semblerait qu'une faune carabique assez diversifiée soit en mesure de s'y développer et donc de permettre une certaine biodiversité entomologique. Ce constat a également été observé en vergers de pommiers cultivés selon le cahier des charges PI et biologique (Vonlanthen et al., 2015).

\section{CONCLUSIONS}

Le désherbage chimique sur la totalité de l'interligne d'une parcelle en production conventionnelle appauvrit considérablement la flore et réduit l'abondance des principales espèces de carabes présentes en vignoble.

En comparaison à des enherbements issus de semis de mélanges d'espèces, un enherbement composé de la flore spontanée favorise l'abondance des carabes et un meilleur équilibre temporel et quantitatif entre les populations des principales espèces.

Les espèces principales des parcelles étudiées ont un régime omnivore (de préférence granivore), mais peuvent exercer une prédation sur certains ravageurs viticoles ayant un stade de développement au sol ou tombant occasionnellement de la vigne ou des adventices.

L'enherbement spontané devrait être privilégié par les viticulteurs pour favoriser les carabes et augmenter leur impact sur les populations de ravageurs.

À l'avenir, des études plus précises relatives aux proies consommées par les espèces présentes en vignobles seraient utiles pour mieux comprendre le rôle régulateur joué par les carabes vis-à-vis des ravageurs de la vigne. Il serait également pertinent de savoir dans quelle mesure certains enherbements viticoles favorisent certains ravageurs tels que les noctuelles qui utilisent les adventices de la vigne.

\section{Remerciements}

Nous tenons particulièrement à remercier Alain Ducommun (Insecta, Neuchâtel) pour sa disponibilité et pour le temps qu'il a consacré à la vérification des identifications de carabes ainsi que Henryk Luka (FiBL, Frick) qui a accepté de confirmer l'identité de certaines espèces problématiques. Merci à Thierry Anet (responsable du vignoble de l'état et canton de Genève) et Rafaël Dunand (viticulteurindépendant) qui ont permis d'effectuer les relevés entomologiques dans leurs vignes et à toutes les personnes qui ont aidé sur le terrain pour la pose et le maintien des pièges.

\section{Bibliographie}

Aeschimann D. \& Burdet H.M., 1994. Flore de la Suisse : le nouveau Binz. Neuchâtel, Suisse : Éditions du Griffon.

Altieri M.A.\& Schmidt L.L., 1985. Cover crop manipulation in northern California orchards and vineyards: effects on arthropod communities. Biol. Agric. Hortic., 3(1), 1-24.

Bohan D.A., Boursault A., Brooks D.R. \& Petit S., 2011. National-scale regulation of the weed seedbank by carabid predators. J. Appl. Ecol., 48(4), 888-898.

Clavien Y. \& Delabays N., 2006. Inventaire floristique des vignes de Suisse romande : connaître la flore pour mieux la gérer. Rev. Suisse Vitic. Arboric. Hortic., 38(6), 335341.

Danne A. et al., 2010. Effects of native grass cover crops on beneficial and pest invertebrates in Australian vineyards. Environ. Entomol., 39(3), 970-978.

Delabays N. et al., 2000. Sélection d'espèces pour l'enherbement des cultures spéciales. Rev. Suisse Vitic. Arboric. Hortic, 32(2), 95-104.

Delabays N., Spring J.-L. \& Mermillod G., 2006. Essai d'enherbement de la vigne avec des espèces peu concurrentielles : aspectsbotaniques etmalherbologiques. Rev. Suisse Vitic. Arboric. Hortic., 38(6), 355-359.

Ehler L.E., 1998. Conservation biological control: past, present, and future. In: Barbosa P.A., ed. Conservation biological control. New York, NY, USA: Academic Press, 1-8.

Fortier E., Shani T. \& Burgos S., 2010. Enherbement du vignoble genevois : bilan et perspectives. Rev. Suisse Vitic. Arboric. Hortic., 42(2), 96-103.

Garcin A., Demarle O. \& Soldati F., 2004. Les carabes, indicateurs de biodiversité et auxiliaires généralistes. Infos CTIFL, 199, 42-47.

Garcin A. \& Mouton S., 2006. Le régime alimentaire des carabes et staphylins. Infos CTIFL, 218, 19-24.

Genini M., 2000. Faune épigée de la vigne et des milieux environnants. Rev. Suisse Vitic. Arboric. Hortic., 32(5), 1-12. 
Goulet H. et al., 2004. Diversity and seasonal activity of ground beetles (Coleoptera: Carabidae) in two vineyards of Southern Quebec, Canada. Ann. Entomol. Soc. Am., 97(6), 1263-1272.

Greenslade P.J.M., 1964. Pitfall trapping as a method for studying populations of Carabidae (Coleoptera). J. Anim. Ecol., 33(2), 301-310.

Hächler M. \& Remund U., 1993. Noctuelles de la vigne. Fiche 612. Nyon, Suisse: Agroscope ChanginsWädenswil, http://www.agroscope.admin .ch/viticultureoenologie/02498/03876/03878/index.html?lang=fr, (04/03/2015).

Heck K.L., van Belle G. \& Simberloff D., 1975. Explicit calculation of the rarefaction diversity measurement and the determination of sufficient sample size. Ecology, 56, 1459-1461.

Holland J.M. \& Luff M.L., 2000. The effects of agricultural practices on Carabidae in temperate agroecosystems. Integr. Pest Manage. Rev., 5(2), 109-129.

Hollander M. \& Wolfe D.A., 1973. Nonparametric statistical methods. New York, NY, USA: John Wiley \& Sons.

Kotze D.J. et al., 2011. Forty years of carabid beetle research in Europe - from taxonomy, biology, ecology and population studies to bioindication, habitat assessment and conservation. ZooKeys, 100, 55-148.

Kromp B., 1999. Carabid beetles in sustainable agriculture: a review on pest control efficacy, cultivation impacts and enhancement. Agric. Ecosyst. Environ., 74, 187-228.

Lauber K., Wagner G. \& Gygax A., 2012. Flora Helvetica. $4^{\text {th }}$ ed. Bern: Haupt.

Levy E.B. \& Madden E.A., 1933. The point method of pasture analysis. N. Z. J. Agric., 46(5), 267-279.

Löbl I. \& Smetana A., eds., 2003. Catalogue of Palaearctic Coleoptera. Vol. 1. Archostemata - Myxophaga Adephaga. Stenstrup, Denmark: Apollo Books.

Lovei G.L. \& Sunderland K.D., 1996. Ecology and behavior of ground beetles (Coleoptera: Carabidae). Annu. Rev. Entomol., 41(1), 231-256.

Luff M.L. \& Turner J., 2007. RES Handbooks for the identification of British insects. Vol. 4. Part 2: The Carabidae (ground beetles) of Britain and Ireland. $2^{\text {nd }}$ ed. Shrewsbury, UK: Field Studies Council.

Luka H. et al., 2009. Carabidae, ecology - Atlas. Fauna Helvetica, 24. Neuchâtel, Suisse : CSCF \& SEG.

Müller-Motzfeld G., ed., 2004. Die Käfer Mitteleuropas, Bd 2: Adephaga. 1. Carabidae (Laufkäfer). Springer Spektrum.

Pasquier D., 2014. Vol des vers de la grappe. Nyon, Suisse : Agroscope Changins-Wädenswil, http://www.db-acw. admin.ch/pubs/vers_grappe_vol.pdf, (23/02/15).

Pétremand G., 2015. Trois espèces de carabes (Coleoptera : Carabidae) sur la liste rouge suisse capturées dans le vignoble du canton de Genève. Entomo Helv., 8, 157160 .
Spring J.-L. \& Delabays N., 2006. Essai d'enherbement de la vigne avec des espèces peu concurrentielles : aspects agronomiques. Rev. Suisse Vitic. Arboric. Hortic., 38(6), 355-359.

Trautner J. \& Geigenmüller K., 1987. Tiger beetles, ground beetles. Illustrated key to the Cicindelidae and Carabidae of Europe. Aichtal, Germany: Josepf Margraf, Publisher.

Trivellone V. et al., 2013. Ragni e carabidi dei vigneti del Cantone Ticino (Svizzera). Boll. Soc. Ticinese Sci. Nat., 101, 63-72.

Vonlanthen O., Fleury D. \& Delabays N., 2015. Influence du mode de production sur les carabes en vergers de pommiers. Rev. Suisse Vitic. Arboric. Hortic., 47(4), 252-258

(33 réf.)

Annexe 1. Espèces présentes dans les mélanges utilisés pour l'enherbement de la vigne - Species present in mixtures used for vineyards ground cover.

\begin{tabular}{ll}
\hline Schweizer Neue $\circledR$ & Schweizer Lenta $®$ \\
\hline Bromus tectorum & Festuca rubra $(60 \%)$ \\
Campanula rotundifolia & Festuca brevipila $(10 \%)$ \\
Dianthus carthusianorum & Poa pratensis $(20 \%)$ \\
Dianthus sylvestris & Poa compressa $(10 \%)$
\end{tabular}

Galium molugo

Helianthemum numularium

Hieracium pilosella

Hypochoeris radicata

Legousia speculum-veneris

Leontodon autumnalis

Lotus corniculatus

Medicago lupulina

Ononis spinosa

Plantago media

Potentilla verna

Primula veris

Prunella grandiflora

Prunella vulgaris

Sanguisorba minor

Sedum acre

Sedum album

Thymus pulegioides

Veronica officinalis

Veronica spicata 